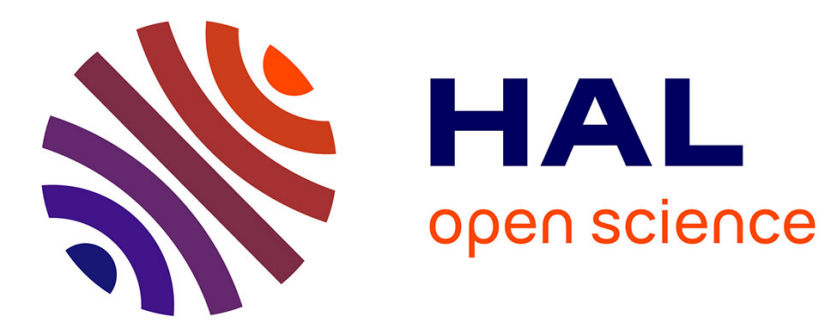

\title{
La sociologie américaine de la pauvreté, du ghetto " wilsonien" à la ville globale
}

Clément Théry, François Bonnet

\section{To cite this version:}

Clément Théry, François Bonnet. La sociologie américaine de la pauvreté, du ghetto "wilsonien " à la ville globale. Sociologie, 2016, 7 (1), pp.77-94. 10.3917/socio.071.0077 . halshs-01308149

\section{HAL Id: halshs-01308149 \\ https://shs.hal.science/halshs-01308149}

Submitted on 27 Apr 2016

HAL is a multi-disciplinary open access archive for the deposit and dissemination of scientific research documents, whether they are published or not. The documents may come from teaching and research institutions in France or abroad, or from public or private research centers.
L'archive ouverte pluridisciplinaire HAL, est destinée au dépôt et à la diffusion de documents scientifiques de niveau recherche, publiés ou non, émanant des établissements d'enseignement et de recherche français ou étrangers, des laboratoires publics ou privés. 


\title{
La sociologie américaine de la pauvreté, du ghetto «wilsonien » à la ville globale
}

\author{
Clément Théry et François Bonnet (CNRS, Pacte)
}

Résumé

Dominante dans le champ académique américain, la sociologie de la pauvreté de William Julius Wilson et de ses héritiers inscrit son objet dans une perspective écologique (ou spatiale). A l'aide des notions de concentration de la pauvreté et d'isolement social, le projet wilsonien est de comprendre la relation entre pauvreté et problèmes sociaux. Fondée sur l'idée de distance d'abord géographique, mais aussi sociale et culturelle, entre quartiers pauvres et noirs et le reste de la société, l'approche wilsonienne est insensible et muette face à une autre configuration spatiale de la pauvreté : celle dans laquelle pauvres et riches vivent dans une grande proximité spatiale, comme c'est le cas dans les villes globales extrêmement inégalitaires. Après avoir déployé la richesse de la sociologie wilsonienne, l'article propose un mode d'analyse complémentaire de la pauvreté. Mettant au cœur de cette analyse la vie économique des pauvres et reléguant à la marge la question des problèmes sociaux, une approche attentive aux effets de la proximité spatiale entre pauvres et riches met au jour non pas les bénéfices d'une mixité sociale qui fluidifierait la circulation des ressources vers les plus pauvres, mais le cloisonnement de ces ressources au travers de relations de dépendance des pauvres envers les riche, rendant élusive une sortie durable de la pauvreté et une intégration dans une « classe moyenne ».

\begin{abstract}
:
Dominant in the field of urban poverty, the work of William Julius Wilson and his followers casts the association between poverty and social problems in an ecological (or spatial) perspective, with concepts like "concentration of poverty" or "social isolation". Grounded in the idea of "distance", spatial distance being concatenated with social and cultural distance, between poor black neighborhoods and the rest of society, the work of Wilson says nothing about another form of urban poverty: one where rich and poor live close to each other, as it is the case in highly unequal global cities in the US. We first unpack the wealth of insights and debates of the "Wilsonian" sociology of poverty, and then develop a complementary mode of analysis that makes sense of poverty in global cities. The core of this latter approach is to put forth the economic life, often off the books, of the poor; to marginalize the issue of the social problems created by the poor; and to highlight the formation of patronage ties between rich and poor. In the end, the image emerging from this second type of urban poverty is one where the benefits of socio-economic diversity in their environment do not trickle down to the poor, as implicitly expected in a "Wilsonian" approach. In a situation of geographical proximity, another form of social closure between poor and rich exists that does not preclude relationships but makes upward mobility for the poor more elusive.
\end{abstract}

Contact

Clément Théry, clement.thery@gmail.com

Mots clefs :

pauvreté ; ghetto ; ville globale ; Etats-Unis ; William Julius Wilson; analyse spatiale ; culture ; communauté ; sociologie urbaine

Keywords :

poverty ; culture ; global city ; United-States; Williams Julius Wilson; spatial analysis ; culture ; community ; urban sociology 


\section{La sociologie américaine de la pauvreté, du ghetto «wilsonien » à la ville globale}

\section{Introduction : le problème de la pauvreté dans la sociologie urbaine américaine}

Depuis les années 1980, aux Etats-Unis, William Julius Wilson exerce une influence hégémonique sur la sociologie de la pauvreté. Son objectif a été de proposer une analyse structurelle des problèmes sociaux pour contrer Losing Ground (1984), l'influente critique conservatrice de l'état-providence de Charles Murray. Dans The Truly Disadvantaged (1987), Wilson explique la pauvreté des inner-cities par la désindustrialisation et la pénurie d'emplois peu qualifiés. Les Noirs se retrouvent confrontés au skills mismatch : ils habitent désormais loin des emplois, se retrouvent au chômage, ce qui dégrade les conditions de vie dans leurs quartiers. Parallèlement, les classes moyennes noires profitent de l'affaiblissement de la ségrégation résidentielle pour fuir ces quartiers, qui deviennent des lieux de concentration de la pauvreté. Cette concentration prive les pauvres de rôles-modèles ${ }^{2}$, diminue le contrôle social informel, modifie la culture des quartiers noirs. Cela produit des modes de vie «pathologiques» (l'expression est de Wilson) que Murray attribuait à l'essor de l'étatprovidence : dépendance aux aides sociales, mères célibataires, trafic de drogue, criminalité. Retenons ici l'idée essentielle : l'association entre pauvreté et problèmes sociaux s'explique chez Wilson par l'impact de forces macro-sociales (transformation de l'économie américaine et migrations) sur les interactions entre individus dans les quartiers pauvres.

L'approche de Wilson est fondamentalement écologique. Cela signifie que la compréhension des mécanismes sociaux est indissociable de leur spatialisation. La spatialisation des processus sociaux s'analyse chez Wilson autour des notions de proximité et de distance, de concentration et de dispersion, d'homogénéité et d'hétérogénéité (Gieryn 2000). Le tour de force de Wilson a été de proposer une approche de la pauvreté qui combine la distance spatiale (enclavement, spatial mismatch), sociale (réseaux déficients) et culturelle (souscultures déviantes) - la première forme de distance induisant les deux suivantes. Cette perspective est devenue une des clés de lecture centrale dans la compréhension de la pauvreté, aussi bien aux Etats-Unis qu'en France, et a justifié les injonctions à la mixité sociale, laquelle devrait favoriser la sortie de la pauvreté pour les plus démunis ${ }^{3}$.

Le programme de recherche de Wilson a inspiré une importante littérature qualitative et ethnographique sur la pauvreté. La thèse de cet article est que cette approche écologique est puissante pour comprendre le lien entre pauvreté et problèmes sociaux dans une configuration où les pauvres sont mis à l'écart, en premier lieu géographiquement, du reste de la société.

\footnotetext{
${ }^{1}$ Nous remercions Loïc Wacquant pour sa lecture critique utile d'une version précédente de ce travail, et Nicolas Duvoux pour ses encouragements et conseils avisés.

2 On traduit role model (modèle positif, exemple à suivre) par «rôle-modèle », afin de faciliter la correspondance avec le langage scientifique anglais.

${ }^{3}$ Le débat qui oppose dans la France des années 2010 Christophe Guilluy à ses détracteurs relève typiquement de l'approche écologique. Pour les uns comme pour les autres, la pauvreté est définie comme la distance aux ressources, les uns insistant sur l'enclavement des banlieues et les discriminations subies par leurs habitants, et les autres sur la distance des populations périurbaines aux richesses des pôles urbaines et leur délaissement par la politique de la ville.
} 
Mais, la littérature wilsonienne est aveugle à une seconde configuration de la pauvreté, celle dans laquelle les pauvres vivent dans une grande proximité spatiale avec les ménages et organisations les plus riches, comme c'est le cas dans la ville globale contemporaine. L'approche de Wilson incite à voir dans cette proximité une opportunité pour l'intégration des pauvres à la classe moyenne. En réalité, en étudiant l'activité économique, souvent informelle, des pauvres dans la ville globale, ce ne sont pas les effets positifs de la mixité sociale qui émergent, mais une multitude de liens de dépendance économique et politique entre riches et pauvres, qui individualisent les destins sociaux, créent des trajectoires de vie en dents de scie, et incitent à une gestion renouvelée des différences statutaires. Dans la proximité entre pauvres et riches propre aux villes globales très inégalitaires, se joue non pas une circulation fluidifiée des ressources matérielles de haut en bas de la pyramide sociale, mais leur cloisonnement, un étagement solidifié dans ces relations de dépendance, rendant élusive une sortie durable de la pauvreté. Nous proposons donc un modèle écologique complémentaire, et non alternatif, à celui de Wilson.

Nous présentons dans la première partie de cet article les textes les plus influents qui ont discuté l'argument écologique de Wilson. Notre premier objectif est de fournir une analyse fine de ce qu'on peut dire sur la pauvreté quand on utilise l'approche de Wilson. Cette partie se centre sur trois thèmes : la nature du lien de communauté dans le ghetto noir (Communauté $1,2,3$ et 4 ), les hypothèses sur le comportement des individus en situation de pauvreté (Individus 1 et 2), et la réintroduction de la culture dans l'explication des problèmes sociaux (Culture 1 et 2). Ces thèmes relèvent d'une littérature de nature essentiellement qualitative, si on omet le travail original et très wilsonien de Rob Sampson (2013), qui mériterait un traitement à part ${ }^{4}$. Cet article ne cherche donc pas à discuter l'œuvre de Wilson en général, ni un certain nombre de débats qui y sont attachés, comme la question de l'underclass ou la culture de la pauvreté. On ne vise pas non plus à montrer les attendus idéologiques d'une certaine sociologie de la pauvreté, comme l'a fait Wacquant $(1997,2002)$.

Dans une seconde partie, l'article entend montrer que la mécanique wilsonienne se révèle inadaptée pour comprendre la pauvreté des villes globales. Prenant appui sur une littérature émergente et centrée davantage sur la ville de New York que sur celle de Chicago, nous analysons les pauvres comme des acteurs économiques prenant part à une économie souvent informelle mais essentielle au dynamisme des grandes villes. Au cœur de cette économie se mettent en place des liens de dépendances entre pauvres et riches, liens fonctionnels pour l'économie des villes globales.

\section{Richesse de l'héritage de Wilson}

\section{Communauté, 1. Disparition et redéfinition du lien de communauté dans le ghetto noir}

On commence cette partie par la question du lien de communauté, et notamment de sa disparition dans le ghetto wilsonien. L'œuvre de Wilson peut se lire comme une lamentation sur la disparition du lien de communauté dans les quartiers noirs, disparition causée la disparition de l'emploi formel et du départ de la classe moyenne noire du ghetto. Le contrôle

\footnotetext{
${ }^{4}$ Pour des revues de littérature de la sociologie qualitative sur la pauvreté, voir Newman et Small (2001) et Newman et Massengill (2006). Pour une adjudication statistique du débat entre Wilson et American Apartheid de Massey et Denton (1993), voir Quillian (1999). Pour un panorama démographique, voir Jargowsky (1997, 2015).
} 
social informel et les obligations mutuelles sont remplacées par anonymat méfiant. Pour Wilson, cette dégradation de la vie collective explique que le ghetto noir concentre aujourd'hui les problèmes sociaux.

Wilson reçoit dès les années 1990 une confirmation de son diagnostic avec le travail statistique de Rob Sampson et de ses coauteurs sur les rapports entre le crime et ce qu'il nomme «collective efficacy ( (voir Sampson 2013). La notion de collective efficacy est une approximation, mesurable à l'aide d'un questionnaire, de l'idée de contrôle social informel, et donc du lien de communauté. La collective efficacy est une propriété du quartier et non des individus : c'est une variable écologique. Il y a une association statistique entre concentration des désavantages économiques et faible collective efficacy. Dans les quartiers noirs et pauvres, quand le lien de communauté disparaît, les problèmes sociaux augmentent.

Sandra Susan Smith montre ainsi dans Lone Pursuit (2007) que l'effondrement du lien de communauté est dommageable pour l'emploi des jeunes hommes noirs peu qualifiés. Smith inscrit son propos dans un questionnement sur le capital social et la confiance interpersonnelle chez les Noirs. Pourquoi est-ce que les immigrés s'entraident sur le marché du travail au point de créer des filières ethniques de recrutement, tandis que les Noirs ont des taux de chômage record ? Smith montre que la confiance est très faible entre Noirs. Les personnes qui ont un emploi valorisent une conception individualiste de leur succès, et ne voient pas pourquoi elles aideraient des gens moins méritants. «Pistonner» un autre Noir comporte un trop grand risque : si le pistonné déçoit, le blâme échoit sur celui qui l'a recommandé. Les appels à la solidarité raciale des hommes au chômage sont donc sans effet. Ce type d'analyse est également présent chez Celeste Watkins-Hayes (2009), qui a étudié les relations entre employés noirs des services sociaux et récipiendaires noirs de l'assistance sociale. Là encore, les appels à la solidarité raciale reçoivent une fin de non-recevoir.

Cependant, l'effondrement des droits et devoirs moraux qui faisaient la vie collective du ghetto selon Wilson est contesté. Dans Off the Books (2006), Venkatesh montre comment l'économie informelle locale, où la violence règle parfois les conflits s'organise sur un principe de réciprocité et non de maximisation du gain individuel. Dans le ghetto, chacun a le droit de faire un peu d'argent pour survivre. La source de cette " économie morale » est à chercher dans l'instabilité des positions qu'occupent les individus. Se plier à cette maxime de réciprocité, c'est se protéger contre les aléas de l'avenir.

Dans «Disposable Ties and the Urban Poor»(2012a), Matthew Desmond décrit un type particulier de lien d'entraide entre les Noirs les plus pauvres, notamment entre les femmes. Ce lien n'est assimilable ni à un «strong tie », ni à une à des relations utilitaires sans solidarité ni confiance. C'est une entraide conditionnelle entre étrangers du ghetto. Après une rencontre à un arrêt de bus ou dans la salle d'attente de l'assistance publique, deux mères célibataires peuvent décider de vivre en colocation, au moins pour quelques mois, et un équilibre délicat s'instaure fondé sur des dons réciproques. Ces individus, qui fréquentent les mêmes lieux et les mêmes institutions, reconnaissent les uns chez les autres des difficultés similaires. La survie de nombreuses personnes noires reposerait sur ces «disposable ties», notamment quand la bonne volonté de la famille et des amis est épuisée.

Sur ce thème, les travaux de Desmond et Venkatesh se démarquent de Wilson, en montrant que le lien de moralité interne au ghetto est le produit immanent des circonstances de vie, et 
non comme l'importation de schèmes extérieurs, issus du mainstream ou de la classe moyenne noire.

\section{Communauté, 2. Retour à Black Metropolis et différenciation dans le ghetto}

L'argument de Wilson repose sur une dynamique historique dont l'état antérieur est analysé par St. Clair Drake et Horace Cayton dans Black Metropolis (1945, réédité par Wilson en 1993). Avant la Deuxième Guerre mondiale, le ghetto rassemblait les Noirs de toute condition: marginaux et ouvriers, femmes de chambres et domestiques, comptables et médecins. L'exemple canonique de ce ghetto est Bronzeville, le quartier noir de Chicago étudié par Drake et Cayton. The Truly Disadvantaged porte sur le même quartier, devenu entre temps un lieu de concentration de la pauvreté..

Black Metropolis soutient trois idées : (a) le ghetto des années 1940 est un monde social complet, différencié et organisé. Les classes moyennes noires ont un devoir moral d'éducation, d'enrichissement et de contrôle paternaliste des couches les plus pauvres du ghetto. Ce sont la presse et les églises noires qui ont la charge de rappeler constamment ce devoir moral et l'impératif de solidarité raciale. (b) Ce monde social en miniature est différent de la société blanche américaine. Dans le ghetto, la moralité est différente, et les institutions ont des rôles abâtardis. Par exemple, le crédit est assuré par les criminels, et non par les banques; le devoir de solidarité raciale supplante la loyauté envers l'Etat et le droit. (c) L'organisation interne du ghetto est structuré par un rapport de dépendance et de domination avec la société blanche ${ }^{5}$. Wilson ne reprend que la première idée de Black Metropolis, le ghetto d'avant-guerre comme monde différencié et organisé. Quand cette hétérogénéité disparaît (la fuite des classes moyennes et le chômage), le ghetto se désorganise, et les problèmes sociaux augmentent. On peut résumer le propos diachronique de Wilson comme le passage de l'organisation dans la différenciation sociale à la désorganisation dans l'identité des positions sociales.

Dans le prolongement critique de Wilson, les ouvrages de Mary Pattillo $(1998,2006)$ et Sudhir Venkatesh $(2000,2006,2008)$ tâchent de réinvestir pleinement Black Metropolis. Pattillo et Venkatesh contestent non seulement l'homogénéisation par le bas du ghetto en excavant une différentiation sociale interne fine, propre au ghetto, mais ils rétablissent aussi les deux idées de Black Metropolis abandonnées par Wilson: ils veulent comprendre comment certains comportements qui apparaissent vus de la société mainstream comme des «problèmes sociaux » sont en réalité le produit de l'ordre social spécifique au ghetto, luimême la conséquence d'une domination économique, sociale et raciale plus large entre noirs et blancs. L'enjeu est de se donner les moyens comprendre la vie en pauvreté avec un regard «depuis » et non «sur » le ghetto, un regard qui normalise les comportements au lieu de les pathologiser, sans effacer la domination raciale dont ces modes de vie sont le produit.

Dans Black Picket Fences (1997), Mary Pattillo s'intéresse, malgré la tendance à la concentration de la pauvreté pointée par Wilson, à la proximité géographique et relationnelle, toujours existante, de la classe moyenne noire avec les Noirs pauvres. Cette proximité distingue la classe moyenne noire de son homologue blanche, et limite ses chances

\footnotetext{
${ }^{5}$ C'est par la référence à Black Metropolis que Wacquant $(1997,2011)$ fournit la définition étroite du ghetto, comme outil de domination raciale historiquement constitué, par opposition à la définition lâche, comme quartier noir et pauvre. Wilson tend à parler d'inner city et de ghetto poor sans faire référence à la définition étroite. Nous suivons son usage.
} 
d'ascension sociale. Contrairement à ce qu'affirme Wilson, l'organisation communautaire qui émerge de cette diversité renforce le crime. Elle peut ralentir la sanction et le contrôle social, parce que le gangbanger (membre d'un gang) est aussi un ami d'enfance, le cousin du pasteur, etc. Dans son ouvrage suivant, Black on the Block (2006), Pattillo s'éloigne des classifications schématiques de la population noire du ghetto en classe moyenne, working class et pauvres. Elle théorise les modalités de différenciation sociale propres au ghetto en rapport avec la subordination du ghetto noir à la société blanche. Ainsi, la position la plus élevée dans cet univers est celle du middle-man, celui ou celle qui vient du ghetto, mais peut jouer l'intermédiaire auprès de la société blanche. Les middle-men (et -women) canalisent les ressources extérieures vers ghetto et choisissent leurs récipiendaires.

Venkatesh décrit une dynamique similaire dans American Project (2000) et Off the Books (2006) avec le concept de broker (courtier) en suivant les échanges qui fonde l'économie informelle du ghetto. Derrière ces échanges, il découvre des positions typiques stables qu'il reconstruit : «la mère de famille », «le pasteur », «l'entrepreneur », «le hustler », «le chef de gang ». Dans ce monde, la maîtrise des ressources venues de l'extérieur est l'élément-clé de la différentiation sociale. Cette approche, à la fois structurale et relationnelle, permet à Venkatesh de reconstruire des logiques de comportement qui apparaissent comme "pathologiques » vue de l'extérieur alors qu'ils sont le produit d'un ordre social local, contraignant pour les individus qui y vivent, mais toujours dépendant et dominé par la société blanche.

\section{Communauté, 3. Faillite des rôles-modèles et dignité}

Dans The Truly Disadvantaged, le contrôle social qu'aurait exercé la classe moyenne noire au temps de Black Metropolis repose sur l'idée de rôle-modèle. La bourgeoisie noire aurait, par son exemple, contenu les problèmes sociaux chez les populations les plus marginalisées du ghetto. C'est cette capacité un peu mystérieuse que Mitchell Duneier conteste dans Slim's Table (1994). Ce faisant, il redirige la sociologie de la pauvreté à l'exact opposé de Wilson, c'est-à-dire loin des thème du ghetto et des problèmes sociaux.

Duneier ne voit dans la peinture historique de Wilson qu'une vision idyllique du ghetto. Il étudie dans Slim's Table (1992) des ouvriers et employés noirs, certains à la retraite, qui habitent dans le ghetto wilsonien, et qui se retrouvent chaque jour dans une cafétéria, à la lisière du ghetto et du quartier de l'Université de Chicago, pour discuter. Eloignés du ghetto aussi bien que de la société banche par leur mode de vie et leur ordre moral, plus traditionalistes que conservateurs, ces ouvriers et employés ne sont des rôles-modèles pour personne.

Cependant, Duneier observe que ces hommes ont gardé une capacité de définir les bases d'une estime de soi et de validation mutuelle qui leur permet de résister aux transformations historiques et de commander respect et déférence malgré les changements historiques qui ont eu lieu autour d'eux. Le problème de sociologie générale qui préoccupe alors Duneier est celui de la dignité des dominés. La dignité est pensée à la croisée du Goffman, très durkheimien, des Rites d'interaction (1967) et des analyses sur les cérémonies de dégradation de Garfinkel (1956). Pour Duneier, la dignité est l'ensemble des rites quotidiens qui rendent hommage au caractère sacré d'un individu dans l'espace public, à sa personne au-delà de sa position sociale, de son genre, de sa race. Duneier fait le constat que ces gestes de déférence 
ne sont pas étendus aux Noirs pauvres et que leur humanité est en suspens dans la société américaine - sans pour autant que cette société apparaisse barbare dans le dépeçage minutieux de l'humanité de ses pauvres.

Dans Sidewalk (1999), Duneier s'interroge sur les conditions de la dignité des populations les plus dominées. Comment cette dignité dans la domination s'élabore-t-elle au quotidien ? Et surtout, comment est-elle in fine refusée par la société dominante, laissant ces individus dans une situation d'invisibilité sociale, de rejet dégouté, voire d'inhumanité ? Duneier étudie des sans-abri noirs qui vendent des magazines usagés dans les rues du quartier bourgeois de Greenwich Village, à New York. Il montre comment l'économie informelle de la vente de magazines ne permet pas seulement à ces hommes de survivre. Elle est aussi la base d'un réinvestissement en soi, une re-sacralisation de sa personne par l'individu lui-même, à l'opposé de l'attitude d'abandon et de dévalorisation que l'auteur appelle la «fuck-it mentality ». Mais cette économie informelle est fragile, et la réinstauration de la dignité des individus qu'elle permet incomplète, car non validée par le reste de la société. Les politiques de la vitre cassée réduisent les ressources, les temps et les espaces, que peuvent s'approprier les sans-domicile-fixe. Ces ressources sont pourtant nécessaires pour alimenter cette économie informelle et pour construire une dignité validée par autrui. Sans ces ressources, ces individus ne peuvent se soustraire au regard des autres. Les sans-domicile-fixe sont forcés par les politiques de la vitre cassée à boire, uriner, dormir, déféquer, se droguer, avoir une vie de famille, en public. Accomplis à la vue de tous, plutôt qu'en privé, ces comportements jettent le doute dans le regard du grand public sur l'humanité de ces hommes noirs et pauvres.

La sociologie de la pauvreté chez Duneier fonctionne sur une tension entre la constitution, d'un côté, d'un ordre économique et moral local dans lequel des individus dominés se redonnent une dignité, et, de l'autre, la déstabilisation de cet ordre par des forces économiques, raciales, policières et urbaines dont l'effet est de déshumaniser ces dominés, de les marginaliser au sens le plus fort - les mettre en marge de l'humanité. Dans On the Run (2014), Alice Goffman, qui a fait sa thèse avec Duneier, reprend ce thème. Elle montre que la réappropriation du rythme de l'institution pénale et judiciaire par les rites de passage des adolescents vers l'âge adulte est imparfaite. Si la première condamnation à la prison permet aux membres d'un jeune couple d'affirmer leur sérieux et leur fidélité l'un envers l'autre, la condamnation reste une cérémonie de dégradation, dans laquelle l'image de soi est tâchée irrémédiablement. La sociologie de la pauvreté de Duneier est une sociologie du marquage symbolique, des gradients de dignité humaine accordée aux individus.

\section{Communauté, 4. Organisations et lien de communauté}

Pour Wilson (1987), la concentration de la pauvreté induit la disparition des ressources institutionnelles locales : disparition des commerces, des associations, des lieux de sociabilité. Les organisations jouent un double rôle : elles fournissent des biens et services, du travail des loisirs. Elles sont aussi le support du contrôle social local et du maintien du lien de communauté dans le ghetto. L'organisation-modèle chez Wilson est l'église noire, qui fournit à la fois les biens du salut, des services matériels, et est un puissant vecteur de contrôle social.

Omar McRoberts étudie dans Streets of Glory (2003) les conditions sous lesquelles certaines églises noires de Boston deviennent des institutions locales, alors que d'autres restent des organisations sans lien avec leur quartier. La variable-clé est la nature de la foi prêchée par le pasteur. Cela implique qu'il ne suffit pas de compter les organisations comme le faisait 
Wilson. Patrick Carr interroge un autre problème : les rapports entre contrôle social informel, organisations locales et contrôles sociaux formels (police et justice). Il étudie un quartier ouvrier blanc de Chicago (Carr 2003). Théoriquement, le contrôle social informel repose sur des relations interpersonnelles denses. Mais dans le quartier de Carr, le contrôle social informel fonctionne grâce aux relations entre les associations locales et les administrations publiques, par exemple dans le cas où une association de quartier fait fermer un bar où se réunissent des petits délinquants.

Mario Small et Nicole Marwell produisent des critiques plus radicales de la place des organisations dans le modèle wilsonien. Small pense le rôle des organisations locales dans les quartiers pauvres à l'aide de la notion de capital social, sans faire référence au contrôle social. Il s'agit de voir comment individus et quartiers pauvres s'accaparent les ressources pour leur survie. Dans Villa Victoria (2004) il montre la concentration de la pauvreté n'a pas eu pour conséquence la spirale infernale wilsonienne : les problèmes sociaux d'un quartier latino de Boston ont été contenus, notamment grâce à une vitalité organisationnelle. Dans Unanticipated Gains (2009), il étudie des femmes pauvres qui mettent leurs enfants dans des crèches (gérées par des associations locales) à New York. Ces crèches sont des espaces de sociabilité où se nouent des relations interpersonnelles qui se révèlent à terme bénéfiques. En d'autres termes, ces organisations ont pour fonction latente de produire du capital social, au sens de connexions avec des ressources, pas de produire du contrôle social.

Cette attention aux organisations et aux ressources auxquelles elles donnent accès, est approfondie par Nicole Marwell (2004, 2007). Marwell travaille sur Williamsburg et Bushwick, à Brooklyn (New York). Comme Small, Marwell échantillonne des quartiers «non-wilsoniens »: pauvres, mais pas dévastés, et pas noirs. Elle se désintéresse des problèmes sociaux. Marwell donne aux organisations un rôle central dans la compréhension de la pauvreté. Les organisations sont des acteurs, avec des logiques propres, dont les décisions sont structurantes dans la vie des pauvres. Comprendre la pauvreté urbaine implique de sortir des schémas où les individus, la famille ou la vie sociale informelle du quartier ont une place centrale. Les sociétés contemporaines reposent sur un réseau dense d'organisations : les organismes de logement social, services sociaux, promoteurs immobiliers, bureaucrates de la police ou de l'état-providence, autorités locales et nationales, etc., doivent être analysés pour comprendre ce qu'il advient, en bout de chaîne, des pauvres.

Marwell s'intéresse particulièrement aux associations locales à but non-lucratif (nonprofits) qui servent de relais pour les politiques sociales. Ces associations répondent à des appels d'offre, reçoivent des financements, et fournissent des services aux pauvres. Marwell étudie donc ces associations, leur rôle concernant le logement, les emplois et la politique. Elle met en évidence comment certaines associations pérennisent leurs financements en incitant leurs personnels et bénéficiaires à voter pour le politicien qui finance lesdites associations, dans un « échange triadique » qui renouvelle la réflexion sur le clientélisme politique (Marwell 2004). L'ambition théorique de Marwell est de renouveler le sempiternel débat entre Chicago et la political economy en sociologie urbaine (McQuarrie et Marwell 2009, Marwell et McQuarrie 2013). Mais elle penche clairement du côté de la political economy : elle invite l'ethnographie urbaine à regarder les questions de pouvoir, les intérêts immobiliers, les puissants technocrates, et à analyser comment ces acteurs comptent bien plus dans la vie des pauvres que les rôles-modèles absents. 


\section{Individus, 1. L'approche interactionniste du ghetto et de ses problèmes sociaux}

Passons maintenant à la question des hypothèses concernant le comportement des individus en situation de pauvreté. On peut lire chez Wilson une conception des individus très proche de celle de l'école de Chicago, ou de Durkheim : il faut que les individus soient intégrés dans des réseaux denses de relations familiales, professionnelles, associatives, etc., pour éviter qu'ils ne soient laissés à eux-mêmes. Sans rôles-modèle, sans institutions, les comportements pathologiques se développent, et l'intégrité de la société est menacée, d'où le concept de «pathologie »: c'est l'anomie, la désorganisation sociale. Il y a donc cette idée que la contrainte, la discipline, l'intériorisation des normes dominantes est positive et désirable, faute de quoi l'ordre social s'effondre. Dans cette section et la suivante, on explore deux façons de penser individu et contrainte dans les ethnographies du ghetto noir pauvre. La première, avec Elijah Anderson, poursuit l'intérêt de Wilson pour les problèmes sociaux, mais théorise la contrainte qu'exerce la société en termes goffmaniens. Son approche est originale, mais sa pente naturelle est de s'éloigner de la problématique de la pauvreté.

Pour les sociologues des interactions comme Erving Goffman ou Randall Collins, les individus investissent beaucoup d'énergie à maintenir des interactions réussies, harmonieuses, où chacun joue son rôle avec succès, ce qui induit de la félicité pour ces individus et reproduit l'ordre social. Les interactions ratées («dysphoriques ») sont donc un objet d'étude privilégié. Anderson étudie deux contextes avec des problèmes potentiels, les interactions entre noirs de classes sociales différentes dans A Place on the Corner (1978) et les interactions entre blancs et noirs dans Streetwise (1990). Le premier livre rend compte d'un café où se retrouvent trois types de clients (noirs) : les regulars (qui ont un emploi mal payé mais stable), les winos (pauvres et un peu alcooliques) et les hoodlums (les racailles, avec une culture oppositionnelle). Anderson ne se contente pas d'observer de la stratification : si ces trois groupes continuent de venir au Café Jelly, c'est parce que les logiques de distinction et d'affirmation relationnelle de statut permettent à chacun d'affirmer une identité propre dans une société blanche qui les voit uniformément comme des noirs du ghetto.

Dans Streetwise (1990), Anderson étudie un quartier de Philadelphie où noirs et blancs cohabitent. La problématique est directement inspirée par l'attention aux problèmes sociaux qui a fait le succès de The Truly Disadvantaged : pourquoi les noirs ont-ils l'air menaçant, indépendamment de leur statut social ? Anderson s'interroge sur la nature de « l'ordre social public » qui prévaut dans les interactions et décrit les règles qui régissent la présentation de soi dans la vie quotidienne d'un quartier mixte. La capacité à lire les situations, la «connaissance de la rue » (streetwise), est un élément important pour des interactions réussies et harmonieuses. La plupart des noirs sont capables de faire la différence entre un homme noir potentiellement dangereux et un homme noir paisible citoyen et bon père de famille qui ne fait qu'accomplir une performance de street credibility pour éviter de devenir une victime. Mais la plupart des blancs ne voient pas la différence, et perçoivent tous les noirs comme potentiellement menaçants. Les hommes noirs sont obligés d'avoir l'air dur pour éviter les ennuis, mais ce faisant ils rendent les blancs nerveux ; et comme les blancs sont bien connus pour ne pas résister en cas d'agression, ils sont plus souvent victimes ; cette dynamique interactionnelle brise rapidement toutes les personnes de bonne volonté.

Dans Code of the Street (1999), le quartier est devenu un ghetto racialement homogène, avec concentration de la pauvreté. Le point le plus notable (dans un livre plus sensationnaliste que goffmanien) est que tout le monde joue son rôle, surdéterminé par les transformations du 
marché du travail. A l'école, les enfants decent jouent aux durs et évitent les bonnes notes, car il faut à tout prix éviter de passer pour blanc (acting white). Les enfants qui ne répondent pas à la violence par la violence se font battre par leurs parents, qui craignent pour le devenir d'un garçon qui ne saurait se défendre lui-même dans le ghetto. En reliant ces logiques de statut à leur encastrement social, Anderson montre les mécanismes interactionnels par lesquels opèrent les changements structurels macro.

Anderson revient aux relations raciales dans The Cosmopolitan Canopy (2011), une ethnographie des espaces quasi-publics interraciaux comme les gares, les centres commerciaux, et le monde du travail. Anderson s'intéresse aux interactions harmonieuses qui célèbrent et performent la société post-raciale mais aussi aux interactions dysphoriques, ce que les noirs appellent les nigger moments, quand parmi toutes ces performances d'harmonie raciale surviennent des interactions où leur statut racial inférieur leur est rappelé. Pareillement, Anderson (2012) montre comment le ghetto porte une ombre toujours présente sur les noirs les mieux intégrés à la société blanche.

\section{Individus, 2. Les trajectoires contraintes}

Loïc Wacquant propose une seconde façon de faire de l'ethnographie des individus en rapport avec des systèmes de contrainte. Au départ, Wacquant s'inscrit assez fidèlement dans la problématique wilsonienne de la nécessité de la discipline pour les individus avec Body and Soul (2004), un livre sur le quotidien des boxeurs d'un club du ghetto de Chicago. Il décrit les jeunes chômeurs du ghetto wilsonien, les enfants de l'ancienne classe ouvrière, des chauffeurs de bus et des facteurs. Cette seconde génération est dans l'impossibilité de reproduire la position sociale des parents. Il n'y a plus de travail autre que l'économie de la drogue et la violence dans la rue (Bourgois 1996). Certains enfants de la classe ouvrière ont cependant conservé l'habitus familial de la discipline au travail. Ils se doivent de trouver une arène dans laquelle investir cette capacité à la discipline, qui puisse leur procurer un statut local et de l'estime, malgré le chômage. Une des rares institutions du ghetto où cet habitus de l'effort et de la discipline peut s'exprimer et être valorisé est le club de boxe. Au gym, ils réaffirment une position sociale supérieure à celle du sous-prolétaire criminel du ghetto, en façonnant une maîtrise de la violence supérieure à celle des autre adolescents et jeunes hommes du quartier. Ceux qui n'ont pas intériorisé l'habitus du sacrifice et du travail, le plus souvent les enfants du sous-prolétariat urbain, s'excluent rapidement du gym.

Mais l'originalité de Wacquant est de proposer une ethnographie où le rapport entre individus et structures est le plus fouillé. Pour Wacquant, la pauvreté dans le ghetto combine différentes instances de domination (économique, raciale, spatiale) qui sont tout simplement trop fortes pour les individus. Faire de l'ethnographie des individus consiste donc à observer et à reconstruire des trajectoires individuelles entre différentes institutions qui semblaient $a$ priori séparées et pour montrer qu'il existe en réalité des connexions déterminantes entre ces institutions - des relations systémiques que nos prénotions nous empêchaient de voir. On retrouve sur ce point l'impératif bourdieusien de construire son objet à rebours des catégories d'Etat et des «problèmes sociaux » (voir aussi Desmond 2014). L'ethnographie sert à suivre des trajectoires individuelles d'individus pauvres qui ne sont pas de hasards. La trajectoire des individus, leur destin social, est compris d'une part comme le passage d'une institution à une autre (la famille, le ghetto, le marché du travail déqualifié et dérégulé, la prison). Ces trajectoires sont des parcours fléchés, dans lequel les expériences et compétences intériorisées issus de la fréquentation d'une institution conditionne, de manière probabilistique, 
l'attachement suivant à telle institution (le club de boxe du ghetto) plutôt qu'à telle autre (la prison). Chaque institution laisse son dépôt en l'individu, sous forme de compétences intériorisées, et détermine plus précisément les alternatives disponibles et les choix probables de l'individu dans le futur, et donc le destin social de la personne. Cette approche est résumée par l'idée de «biographical-cum-institutional data »(Wacquant 2002, p. 1480).

Ainsi, à partir du début des années 2000, Wacquant théorise un lien de nature systémique entre le ghetto noir wilsonien, le système pénal qui vise les noirs en priorité, un marché de l'emploi déqualifié extrêmement précarisé, un Etat-providence qui se retire, et une exclusion des jeunes noirs de la sphère politique (Wacquant 2001 ; Wacquant 2009). Ce qu'apporte l'ethnographie, en propre, à la sociologie du ghetto avec ces «institutional-cum-biographical data », c'est une documentation des liens fonctionnels entre des sphères institutionnelles que l'on croyait indépendantes : le ghetto prépare les jeunes noirs aux allers-retours permanent entre un marché de l'emploi très précarisé et la prison, menant à une réduction drastique des droits civiques et sociaux des individus (Pettit et Western 2004). Il y aurait des «pipelines » entre ces institutions, les individus se déversant quasi mécaniquement de l'une vers l'autre.

On retrouve une conception voisine des relations ghetto noir-système pénal chez Alice Goffman dans On the Run (2014), avec une attention particulière pour le pouvoir de l'état policier dans la vie du ghetto. Dans The Stickup Kids (2012), Randol Contreras examine les trajectoires des braqueurs de dealers de drogue. Leur travail consiste à torturer des dealers pour obtenir la drogue et/ou l'argent. Contreras comprend le phénomène comme l'interaction entre d'un côté des structures (des hommes pauvres au chômage, les transformations du marché de la drogue dans les années 1990) et des trajectoires où les individus finissent en prison (notamment à cause de l'extension de l'Etat carcéral) et y vivent une socialisation à l'hyper-violence qui leur ouvre la carrière de braqueurs de dealer (voir le compte-rendu dans ce numéro). Contreras construit son argumentation comme les étapes d'un cycle de vie.

Matthew Desmond complète ce tableau dans ses études ethnographiques et multi-méthodes sur les expulsions locatives des femmes noires (Desmond 2012b, Desmond et Valdez 2013). Pour les jeunes hommes noirs, le tandem ghetto-prison est l'expérience sociale par laquelle se crée une main d'œuvre docile et sans pouvoir politique; pour les femmes noires, cette fonction de domination est remplie par l'expulsion pour loyer impayé. L'expulsion est au cycle de vie des femmes noires ce que la prison est à celui des hommes : une étape déterminante dans l'acquisition d'un master-status négatif, qui limite considérablement les chances de vie des femmes noires du ghetto, et imprime une inflexion décisive sur leur destin.

Wacquant et son héritage font une ethnographie du ghetto où la contrainte structurelle est au cœur de la description ethnographique des individus la plus détaillée. Ce faisant, ils s'éloignent du schéma wilsonien, et de sa conception désorganisation sociale / nécessité du contrôle social. Pour Desmond par exemple, c'est l'exploitation qui engendre la pauvreté. Wacquant étudie le ghetto wilsonien en rapport avec les autres institutions de domination des noirs aussi bien synchroniquement (c'est-à-dire en lien avec la prison, le marché du travail etc.) que diachroniquement (c'est-à-dire comme la modalité présente de domination raciale après l'esclavage et Jim Crow). Alors que le ghetto wilsonien semblait le produits conjoncturel de plusieurs forces macro-structurelles indépendantes, le ghetto d'ethnographes 
comme Wacquant et Desmond est l'un des rouages essentiels d'un système plus large et complexe de domination raciale dans la société américaine.

\section{Culture, 1. Le quartier comme médiateur entre structure et culture}

L'approche culturaliste de la sociologie de la pauvreté a longtemps constitué un repoussoir (Duvoux 2010). L'apport spécifique de Wilson est de faire de la culture une variable écologique. Pour Wilson, le quartier (la variable écologique) fonctionne comme un opérateur entre les forces macro-sociales (la désindustrialisation, la fuite des classes moyennes noires) et la culture des individus. L'existence de problèmes sociaux comme le crime, la sexualité sans obligation familiale, et le non-respect des institutions sont compris comme le résultat d'une culture locale non seulement déficiente (en valeurs), mais aussi inefficace, c'est-à-dire ne remplissant pas sa fonction d'intégration sociale. Le concept de culture tend donc chez Wilson vers la notion de contrôle social de l'école de Chicago.

Au cours des années 2000, plusieurs auteurs entreprennent de renouveler la pensée de Wilson. Trois questions guident cette littérature. La première porte sur la définition de la culture. Les approches post-Wilson utilisent les concepts de la sociologie contemporaine de la culture : les cadres (frames), les répertoires (repertoires), les récits (narratives), des frontières morales (symbolic boundaries), etc. (Lamont et Small 2008). Ainsi, dans Villa Victoria (2004), Mario Small analyse l'évolution intergénérationnelle du rapport au quartier en termes de frames : la génération précédente percevait le quartier à travers le frame de l'action collective, du bien public à défendre, tandis que la génération suivante a abandonné ce frame pour considérer Villa Victoria comme un simple quartier latino pauvre.

La deuxième question porte sur la relation entre quartier et culture, et plus précisément sur les processus sociaux qui lient un aspect particulier de la culture d'un individu à une variable de son environnement immédiat. Par exemple, Alford Young étudie ce qui façonne les aspirations à la mobilité sociale de 26 hommes noirs pauvres du ghetto de Chicago dans The Minds of Marginalized Young Men (2004). Les interviewés se partagent en deux catégories, en fonction du rapport au quartier. Les uns vivent toute leur vie dans le ghetto, en situation d'isolement social ; les autres ont plus de contacts avec l'extérieur, parfois grâce à leur travail, souvent à cause de la prison. Plus les hommes ont des contacts avec le monde extérieur, mieux ils comprennent que leurs chances de mobilité sont nulles, et mieux ils réalisent que leur vie sera dure jusqu'à la fin. Inversement, plus ils sont isolés, moins ils comprennent la réalité objective de leurs chances de vie, et plus ils se font des idées irréalistes sur leur futur. Le rapport au quartier influe sur les schèmes de perception et les aspirations : en d'autres termes, la variable écologique agit sur la variable culturelle.

La troisième question porte sur le problème de l'approche culturelle dans les quartiers hétérogènes. Wilson postule des ghettos homogènes, où la transmission culturelle est mécanique. Mais dans la réalité urbaine des années 2000, il y a très peu de ghettos homogènes (on y reviendra). Comment conjuguer l'approche écologique de la culture des quartiers pauvres avec l'hétérogénéité sociale de ces quartiers ? C'est le propos de David Harding dans Living the Drama (2010). Il se demande pourquoi les jeunes noirs de 10-11 ans peuvent avoir une image aussi négative de la police, alors qu'ils n'interagissent presque jamais avec des policiers. Comment se transmet l'expérience des aînés ? Le quartier rassemble des enfants et des adolescents qui ne vont pas à l'école et qui traînent dans la rue, et des adultes au chômage qui traînent aussi dans la rue. Les interactions entre les uns et les autres permettent la 
transmission des valeurs culturelles particulières au ghetto. Par exemple, il est inutile de travailler à l'école puisque les noirs sont systématiquement discriminés sur le marché du travail, et il faut sublimer la faillite de la masculinité comme breadwinner avec une sexualité hypersexiste. Harding recoure notamment à la notion «d'écho culturel » comme mécanisme de transmission: pour faire comprendre aux enfants que les policiers sont maléfiques, les adultes s'appuient sur la comparaison avec les professeurs à l'école, forcément injustes, arbitraires et punitifs envers les enfants en situation d'échec. Ainsi s'apprend le «cynisme légal » (legal cynicism), la méfiance systématique à l'égard de la justice et de la police.

Dans ces travaux, et pour utiliser un vocabulaire un peu schématique, l'espace est la variable intermédiaire entre structure et culture. C'est la proximité spatiale qui permet à du contexte structurel de façonner les schèmes culturels. La logique de cet argument est intégralement wilsonienne, puisque toutes les variables-clés s'y retrouvent : la concentration de la pauvreté (l'espace), la réalité économique (la structure) et la (sous-)culture du ghetto.

\section{Culture, 2. Problèmes de valeurs, ou problème d'argent?}

Le travail de Kathryn Edin et de ses coauteures constitue une réfutation systématique de l'argument selon lequel les pauvres auraient une culture / des valeurs / des frames différents. Edin montre que les comportements pathologiques procèdent de l'impossibilité matérielle d'agir en cohérence avec les valeurs mainstream. Edin fait un argument mertonien, similaire à la démarche d'Eliott Liebow dans Tally's Corner (1967).

Pour démontrer que les valeurs sont largement partagées, Edin échantillonne des pauvres blancs, noirs et latinos, avec un double critère de revenu et de comportement soi-disant pathologique. Dans Makind Ends Meet (1997), Edin et Lein interviewent 379 mères célibataires pauvres. Toutes les mères de l'échantillon (sauf une) voudraient travailler, ont pleine conscience des normes en vigueur dans le monde du travail, ne considèrent pas les aides sociales comme une solution satisfaisante, et veulent toutes être des bonnes mères qui s'occupent bien de leurs enfants (pp. 7-8 et 190). Dans Promises I Can Keep (2005), Edin et Kefalas interviewent 162 mères célibataires pauvres afin de comprendre les raisons et les mécanismes des naissances hors-mariages (un déterminant très sûr aux Etats-Unis de la pauvreté de la mère et de ses enfants). Elles montrent que les mères pauvres ont des valeurs parfaitement mainstream sur le mariage, la famille et les enfants. C'est parce que la réalité économique et sociale ne peut pas permettre des mariages à la hauteur des attentes des femmes que celles-ci ne se marient pas. Les femmes interviewées identifient le mariage au couronnement de l'ascension sociale : une vie économiquement stable, protégée des difficultés matérielles, un statut de propriétaire, bref, tout ce que le chômage, l'alcoolisme, la drogue, et la petite criminalité viennent contrecarrer. Comme les femmes des classes moyennes, les femmes pauvres dans les sociétés contemporaines n'ont plus obligation sociale de se marier (pour avoir accès au sexe et à une forme minimale de légitimité comme individu). Du coup, elles refusent les maris que leurs grand-mères ont accepté à l'époque où tout le monde se mariait. Le point important ici est la similitude des valeurs à travers les classes sociales.

Le travail de Kathryn Edin invite à une discussion de fond sur ce qu'est la pauvreté. Au plus simple, Edin définit la pauvreté comme le manque d'argent. C'est tout le propos de Making Ends Meet : dans la plupart des cas, les emplois accessibles pour les femmes non-qualifiées ne sont pas assez rémunérateurs et impliquent des coûts (de transport et de garde d'enfants) qui 
rendent le travail moins attractif qu'une combinaison d'aides sociales et d'économie informelle. Etre pauvre, c'est ne pas avoir assez d'argent, en subir les conséquences (avoir faim, froid, se faire couper l'électricité, être expulsée de son logement), et chercher à se prémunir du manque (en recourant à l'économie informelle, en recevant des prêts et des cadeaux de la famille et des amis, et en vivant chez ses parents). Edin et Kefalas font un raisonnement analogue dans Promises I Can Keep : au final, ce n'est pas tant avoir un enfant tôt et hors-mariage qui est si nuisible pour les chances de vie : c'est d'avoir des chances de vie aussi faibles au départ qui conduisent les femmes à avoir un enfant très tôt, puisque c'est la seule chose qu'elles peuvent faire qui leur apporte de l'estime de soi. Conclusion qui n'est pas si triviale qu'elle n'en a l'air : si les pauvres avaient accès sur le marché du travail à des emplois sûrs et bien payés, ils ne seraient pas pauvres (voir également la recension de It's Not Like I'm Poor [2015], écrit avec Sarah Halpern-Meekin, Laura Tach et Jennifer Sykes, dans ce numéro).

\section{Au-delà de Chicago : vie économique et les liens de dépendance dans la ville globale}

\section{Les limites de l'approche wilsonienne de la pauvreté}

Nous avons jusqu'ici souligné la richesse de l'approche écologique de Wilson. Nous allons maintenant montrer deux limites de cette approche. La première limite est celle de ses concepts centraux : culture, contrôle social informel, rôles-modèles. Ces concepts laissent peu de place à une analyse en termes de pouvoir, de rapports de force, de domination, voire d'exploitation. C'est notamment le sens du travail de Wacquant et Desmond que de problématiser la question des liens interpersonnels dans le ghetto au travers de questionnements plus conflictualistes. Quand Wilson revendique un approche «structurelle », il fait référence au marché du travail et aux migrations, mais il ne problématise ni l'Etat, ni le capitalisme.

La seconde limite tient au domaine de validité géographique du propos wilsonien. Plusieurs auteurs que nous avons discuté sont sortis du ghetto noir de Chicago et ont développé des approches en rupture avec Wilson. Quand Kathryn Edin compare des pauvres noirs, blancs et latinos à travers les Etats-Unis, elle ne voit pas de différence culturelle. Quand Nicole Marwell étudie des quartiers juifs et latinos à New York, elle est plus intéressée par les rapports de pouvoir entre organisations locales et autorités publiques que par les problèmes sociaux. Se pose donc la question de la spécificité du ghetto noir de Chicago, qui est le fondement empirique de The Truly Disadvantaged et de nombre d'ethnographies des étudiants de Wilson. Une façon de trancher cette question est de compter le nombre de quartiers noirs, pauvres, dont la population décroît et avec une faible densité organisationnelle. Avec des critères wilsoniens, il n'y a dans tous les Etats-Unis urbains que 9 « ghettos » sur 13555 quartiers (Small 2008). On ne peut donc résumer la pauvreté urbaine au ghetto wilsonien.

Ainsi, ce n'est pas un hasard si Marwell étudie New York, et non Chicago. Pour l'auteur, dans les villes globales comme New York où les ressources institutionnelles sont abondantes, le problème n'est pas celui d'individus laissés à eux-mêmes, mais celui des rapports de pouvoir entre les différents niveaux d'acteurs politiques, les acteurs philanthropiques, les capitalistes (notamment les promoteurs immobiliers), les mouvements sociaux locaux et les grandes bureaucraties (administrations, police). De fait, il semble impossible aujourd'hui d'étudier la pauvreté sans mentionner le rôle changeant de l'état-providence après la welfare reform de 
1996 et sans prendre en compte l'incarcération de masse et les évolutions technologiques des appareils policiers (Allard et Small 2013). On s'éloigne fortement du contrôle social informel de Chicago.

Cela nous guide vers l'idée que le modèle wilsonien est spécifique à la réalité du South Side de Chicago dans les années 1980-1990. Ce ghetto ne serait qu'une configuration particulière de pauvreté urbaine, pas forcément la plus représentative ni la plus stratégique (Small 2008). La spécificité de Chicago serait d'offrir un cas de pauvreté (noire) séparée du reste de la société, isolée spatialement, où les (noirs) pauvres sont coupés des ressources et des emplois. Loin de cette tradition, des auteurs d'inspiration marxiste ont théorisé dans les années 1980 l'émergence de la ville globale, dans laquelle les populations pauvres vivent dans un environnement urbain marqué par des inégalités économiques extrêmes (Sassen 1991, Mollenkopf et Castells 1991). La proximité spatiale et les contacts directs et récurrents entre individus pauvres et entités riches (individus ou organisations) rendent les analyses issues de Wilson inopérantes dans la ville globale. Comment analyser la pauvreté de la ville globale ? Faut-il abandonner l'approche écologique pour comprendre ce que c'est qu'être pauvre dans la ville globale ? On reprend ici les trois thématiques de la première partie (individus, culture, lien social) et on montre comment elles se déploient dans cette forme urbaine.

\section{Individus : les pauvres comme acteurs économiques}

Chez Wilson, les pauvres sont des fauteurs potentiels de problèmes sociaux. Leur activité économique quotidienne est considérée par hypothèse comme marginale, et sans intérêt. Dans la ville globale, les pauvres sont au contraire à analyser comme des acteurs économiques : la proximité spatiale avec les ressources permet le développement d'une économie fondée sur les relations entre pauvres et riches. Le programme de recherche qui découle de ce constat vise à élucider la dynamique de ces circuits économiques, ainsi que les conséquences de la participation à ces circuits pour les inégalités et les trajectoires individuelles. Cette sociologie de la pauvreté de la ville globale implique de replacer les relations économiques des pauvres dans le cadre d'une économie politique plus vaste où les notions d'exploitation et de dépendance sont centrales.

Dans la perspective de la ville globale, trois champs de recherche secondaires chez Wilson deviennent stratégiques. Le premier concerne les rapports et échanges entre économie formelle et économie informelle et s'appuie sur la sociologie économique de l'immigration (Light et Bonacich 1988, Portes et Sensenbrenner 1993, Waldinger 1996, Waldinger et Lichter 2003). Cette littérature tend à donner l'impression que l'économie informelle est la prérogative des immigrés. Il faut au contraire étudier la participation de tous les types de pauvres, ainsi que des classes moyennes et supérieures, à l'économie informelle, ce qu'essaie de faire par exemple Venkatesh $(2006,2013)$. Ensuite, la littérature sur l'immigration a tendance à réifier les identités ethniques et raciales dans le but d'expliquer les phénomènes de solidarité économique et d'exploitation consentie, avec notamment la notion d'enclave ethnique (Zhou et Logan 1989). Pour la sociologie de la pauvreté de la ville globale, les identités ethno-raciales sont moins des outils de fermeture du groupe sur lui-même que des frontières manipulées dans le but de créer, maintenir et étendre des relations économiques (voir Lee 2002 pour un travail précurseur sur ce sujet). Ces identités «dures » n'étant plus disponibles comme la base de la solidarité et de l'acceptation de l'exploitation, on doit s'interroger sur les modalités d'instauration de la confiance et de régulation du conflit, avec des auteurs comme Venkatesh (2006, 2013) Duneier (1999), et Desmond (2012a). Enfin, il 
faut comprendre la nature de la relation qui unit l'économie formelle et informelle. Trois modèles existent aujourd'hui dans la littérature. Le premier est celui de la ressource résiduelle et du parasite : l'économie informelle, et les pauvres qui y participent, subsistent grâce au trop-plein de ressources de l'économie formelle. C'est le cas des revendeurs de journaux usagers de Duneier (1999), ou des recycleurs de déchets de Gowan (2010). Le second modèle, après Sassen et les fonctions de la pauvreté d'Herbert Gans (1972), est celui de la relation fonctionnelle. Il s'agit d'analyser comment l'économie formelle prend appui, souvent en le niant, sur l'économie informelle pour augmenter ses profits et maximiser sa flexibilité. Le troisième modèle est l'hybridation entre formel et informel: les tentatives d'Uber pour le secteur du taxi, ou d'AirBnB pour l'industrie hôtelière pour «informaliser » le travail protégé mais peu qualifié participe de la création d'une zone économique intermédiaire, au statut légal disputé, dans laquelle une part croissante des pauvres des villes globales est impliquée. En résumé, notre première grande hypothèse de travail est que la proximité spatiale entre riches et pauvres permet des arrangements économiques entre ces deux populations qui défient les approches en termes de marché pur et formel, et en termes d'enclave ethnique repliée sur ellemême. En étant attentif à la dimension informelle de ces circuits, on se donne les moyens de révéler l'activité économique des pauvres comme partie prenante de l'économie des villes globale.

Le deuxième champ de recherche d'une sociologie de la pauvreté qui s'intéresse à l'activité économique des pauvres consiste à étudier les trajectoires des individus et la production des inégalités par la participation aux circuits économiques locaux. La sociologie du ghetto wilsonien s'est peu intéressée (à part chez Wacquant) aux trajectoires individuelles, parce que le ghetto est compris comme une trappe à pauvreté, un lieu de stase sociale. Dans les villes globales, en revanche, comme décrit par Katherine Newman et Victor Chen dans The Missing Class (Newman et Chen 2007), les trajectoires des individus et des familles pauvres sont étonnantes de retournements de fortune et d'amplitude vers le haut et vers le bas. Une famille peut jouir d'un mode de vie de classe moyenne pour quelques années avant de (re)tomber dans la grande pauvreté (voir aussi Black 2009 pour une étude longitudinale d'une famille Latino). L'enjeu est d'analyser les règles et probabilité d'ascension dans cette économie à la croisée du formel et de l'informel, ses plafonds, et les forces de retombée. Pour Newman et Chen, l'instabilité matérielle est le propre de cette «missing class», située entre les pauvres du ghetto wilsonien et la classe ouvrière stabilisée. Pour les auteurs, cette instabilité est le produit d'accidents que le système de protection sociale américain rend impondérables et fatals (maladie, divorce, perte d'emploi). Floating City de Venkatesh (2013) est aussi construit autour de cette problématique, mais avec moins d'intérêt sur la notion de groupe social, de «class». L'auteur s'intéresse à l'exploitation interpersonnelle des pauvres par les riches dans l'économie de la ville globale, c'est-à-dire aux trajectoires économiques de vendeurs de vidéos pornographiques, de prostituées, de proxénètes et de dealers de drogue (l'exploitation est également au centre du travail de Desmond). Venkatesh découvre les particularités de la ville globale (et, en ricochet, le particularisme de Chicago) : " la clé du succès [à New York] était le talent d'utiliser et d'abandonner des relations improvisées » (p. 270). Pour comprendre les inégalités qui émergent dans cette économie particulière, Venkatesh pointe les soft skills, les compétences sociales qui permettent à des individus de gagner de l'argent en faisant du commerce à travers les barrières sociales. Une telle idée était déjà présente chez Wilson (1996), mais elle devient centrale pour l'analyse de la vie économique des pauvres dans la ville globale. 
Derrière la mise en avant de trajectoires-types se dessine une redéfinition non seulement de la notion de pauvreté, mais aussi des frontières et de la dynamique de formation du groupe social des «pauvres ». S'intéresser à la vie économique des pauvres, c'est concevoir la pauvreté en termes de revenu et d'instabilité du revenu. Suivant Venkatesh, Newman et Black, être pauvre n'est pas tant le fait de gagner en dessous d'un certain montant; c'est l'impossibilité de se mettre à l'abri durablement du risque de gagner en dessous de ce revenu référent. Ainsi, la mesure qu'utilisent les sociologues wilsoniens (un quartier est pauvre lorsqu'à un moment donné, $40 \%$ de sa population gagne moins de $80 \%$ du revenu médian de l'aire métropolitaine) reflète mal les spécificités de la pauvreté dans la ville globale: proximité entre riches et pauvres, d'un côté, et trajectoires économiques erratiques des ménages les plus démunis, de l'autre. Etre sensible à cette réalité propre aux villes globales permet de nouvelles hypothèses sur la dynamique de formation des groupes sociaux dominés. Par exemple, le phénomène de gentrification peut-être relu, non seulement comme déplacement des pauvres par une dynamique d'inégalité croissante dans la ville globale, mais également comme déformation du groupe social des plus pauvres. L'instabilité des trajectoires des pauvres, vers le haut et vers le bas d'un côté, et précarisation des classes moyennes de l'autre suggèrent des passerelles et fusion entre gentrifieurs et gentrifiés. Ces passerelles qui peuvent être faites d'échanges économiques, de l'émergence de modes de vie compatibles entre des groupes par ailleurs en lutte, voire de liens matrimoniaux. La gentrification ne serait donc pas seulement une dynamique spatiale, mais également une dynamique morphologique, c'est-à-dire la constitution d'un groupe social produit de l'agglomération des tranches inférieures des classes moyennes précarisées que sont certains gentrifieurs, aux pauvres insérés dans les circuits économiques instables de la ville globale, habituellement du côté des gentrifiés.

Enfin, placer au centre de l'analyse des pauvres l'activité économique invite à un troisième champ de recherche : une relecture de l'expérience subjective de la pauvreté (Newman et Massengill 2006). Dans le ghetto wilsonien, cette expérience est marquée soit par l'aspiration irréaliste à un avenir meilleur (Young 2004), soit par la dépression sous le poids des contraintes (Contreras 2012). Dans la ville globale, cette expérience est celle de la frustration et du ressentiment, oscillation entre dépit et optimisme, les richesses semblant à la fois à portée de mains, et, en réalité, en partie cachées et inaccessibles car ne pouvant être saisies que par l'insertion dans des relations de dépendance, souvent instables et humiliantes, propres à l'économie partiellement informelle de la ville globale (Thery 2014).

\section{Du lien de communauté aux liens de dépendance}

Chez Wilson, la disparition du lien de communauté permet de comprendre la relation entre pauvreté, ghetto et problèmes sociaux. Dans la ville globale, le lien social à étudier est celui de dépendance, de patronage ou clientélisme, entre les riches et les personnes sans ressource. La proximité spatiale entre les personnes aux ressources immenses et les pauvres permet la création d'un type d'échange calqué sur les relations entre patrons et clients (et non un échange marchand ou une relation bureaucratique).

Une relation de patronage (ou de clientèle) est un lien sur lequel circule des dons réciproques entre deux personnes de statut économique et social opposé. Les valeurs des biens et services qui circulent sur cette relation sont difficilement mesurables et comparables, d'autant que cette circulation a lieu selon une temporalité distendue. L'objet de ces relations, en outre, n'est pas restreint : c'est l'entièreté de la personne la plus démunie qui peut être appelée à agir 
dans le cadre de la relation de patronage : c'est donc une relation hautement personnelle. Ces relations se manifestent sous la forme de services mutuellement donnés et rendus, bien qu'il s'agisse, en réalité, de relations d'échanges utilitaires, dont les coûts et les avantages sont finement calculés et qui nécessitent que chacune des parties y trouve son compte pour perdurer. Wolf parle à leur propos, «d'amitiés déformées » («lopsided friendship », Wolf 1966). Ces relations déjouent à la fois le formalisme des relations bureaucratiques et de marché (Boissevain 1966). Dans la littérature sur la pauvreté aux Etats-Unis, la relation de patronage apparaît d'abord comme une chose du passé. Elle est décrite dans les relations entre hommes politiques locaux et populations immigrées, souvent venues d'Europe avant la seconde guerre mondiale. On la retrouve ainsi dans la seconde partie de Street Corner Society de Whyte (1943). La littérature sur les «machines politiques »dans les villes américaines est également pleine de relations de patronage, dans lesquelles votes et loyauté politique s'échangent contre contrats publics, emplois municipaux et assistance sociale (Bonnet 2010, Erie 1990).

Une lecture fine de la littérature qualitative contemporaine sur la pauvreté témoigne de la persistance de ce type de lien dans les quartiers pauvres des villes contemporaines - et spécifiquement dans la ville globale. Dans Off the Books, Venkatesh décrit l'ensemble des services, du nettoyage aux actes sexuels, que fournissent les femmes du ghetto au pasteur, apparemment gratuitement. Elles espèrent en fait obtenir un jour un emploi de baby-sitter ou d'aide ménagère dans une famille blanche d'un quartier aisé. Dans Bargaining for Brooklyn de Marwell, les ONG locales demandent à leurs clients, à qui elles fournissent des services sociaux, de participer aux meetings politiques, aux manifestations et de voter pour les candidats qui octroient des subventions aux ONG en question; c'est «l'échange triadique ». Dans une étude ethnographique sur le marché immobilier des quartiers pauvres de Brooklyn, Clément Thery montre que la vaste économie informelle de ce secteur, souvent de nature prédatrice, repose sur des liens de patronage entre des acteurs différents, certains pauvres et certains extrêmement riches. L'efficacité de ces liens dans la poursuite de gains économiques repose sur le fait d'aller à l'encontre de l'organisation officielle et formelle du marché immobilier. Les liens de patronage permettent aux individus de développer des stratégies économiques non prévues par le marché : l'individu le plus riche en ressources utilise l'individu le plus démuni dans un jeu économique complexe que lui seul maîtrise, et dans lequel l'individu le plus pauvre n'est qu'un pion, mais un pion volontaire parce qu'il y trouve des ressources et l'expérience d'une vie économique active (Thery 2014). Dans les liens de patronage propres à l'économie informelle des villes globales se dessinent pour les individus les plus pauvres les voies d'accès à des ressources, voire à une ascension sociale limitée, même si elle se révèle plus transitoire que durable.

Dans cet environnement économique, fait de proximité avec des richesses énormes et de liens de dépendance interpersonnelle, les plus pauvres passent leur temps à faire le bilan économique de leurs relations, de leurs alliances latérales et verticales, à se méfier des autres individus qui pourraient les exploiter en leur faisant croire que leurs intérêts sont alignés. C'est une économie profondément épuisante et interpersonnelle, émotionnelle et arbitraire, bien plus que régulière. S'esquisse ici un processus de dé-rationalisation de l'économie, après des décennies de rationalisation sous la pression de la bureaucratisation de la vie économique et la défense des droits économiques et sociaux des pauvres (Katz 1982). 


\section{Culture : de la sous-culture au management du statut}

Pour la sociologie des pauvres de la ville globale, la culture et les valeurs ne sont pas des problématiques centrales. Il ne s'agit pas de savoir si les pauvres développent une sousculture en opposition à la classe moyenne. Au contraire, dans un monde de proximité spatiale, le problème est celui du management du statut, dans un sens goffmanien : comment se mettent en scène, se préservent, mais aussi se subvertissent, les différences de statut énormes dans les contacts quotidiens entre pauvres et riches?

Cette gestion de la distance et du statut dans la ville globale est explorée dans Doormen (2005) de Peter Bearman. Les doormen sont les concierges-portiers des immeubles huppés de New York. Leur principale fonction est de gérer l'accès à l'immeuble, pour empêcher les intrusions de cambrioleurs, démarcheurs, mendiants, etc. Bearman s'intéresse au contact presque intime entre des individus de statuts sociaux opposés. Les doormen ont accès à une partie significative de la vie privée des habitants de l'immeuble: les secrets honteux, les habitudes étranges, tout ce qui est en décalage avec la décence et l'honneur upper-class. Cette connaissance intime est potentiellement dangereuse pour le statut des classes supérieures. Mais Bearman montre comment le désir de professionnalisation des doormen et le statut des habitants upper-class des immeubles se renforcent mutuellement - neutralisant la dangerosité du contact intime. Pour les doormen les plus habiles, le contact intime est l'occasion de développer une connaissance des besoins personnels des habitants, connaissance parfois tacite et complice, et d'orner ainsi leur travail de la rhétorique de la professionnalisation. Pour les résidents, recevoir un service personnalisé, et donc fondé sur une connaissance intime de leur vie, est la marque de l'unicité de leur personne, de leur valeur interne par rapport aux voisins et au reste des classes supérieures.

Le jeu à somme positive entre statut des doormen et statut des classes supérieures n'a pas nécessairement lieu. Beaucoup de doormen se voient comme des serviteurs et développent une haine tenace de leurs résidents, des privilégiés incapables de changer une ampoule, justes bons à hériter la fortune de la famille, et qui les méprisent. Ces doormen échouent à gérer la distance sociale avec les occupants fortunés, les interactions sont désagréables et maladroites, et ils se font licencier. Pour éviter cette fin, ils mettent en place des rituels hypocrites, décrits par James Scott dans Weapons of the Weak (1985), faits de sourires de façade, de cérémoniels subreptices de dégradation des classes supérieures, de gestes de courtoisie ironiques et à double-sens. Par ces pratiques, les pauvres réactivent leur dignité contre les riches (sans grande portée, voir Duneier 1999), tout en préservant leur intérêt économique.

$$
* * *
$$

En résumé, être pauvre dans la ville globale, c'est habiter un contexte urbain qui déborde de richesses et qui est marqué par des inégalités immenses. Ce n'est pas la même chose qu'être pauvre dans l'environnement dévasté du ghetto wilsonien. L'activité économique des pauvres wilsoniens est presque sans intérêt dans un contexte intellectuel et politique où la question fondamentale est celle des problèmes sociaux générés par les pauvres. Dans la ville globale, la pauvreté est avant tout liée au fait d'avoir une activité économique pressante et usante, instable et interpersonnelle, et souvent humiliante, dans le cadre de relations de dépendance. C'est au travers de ces activités et ces relations que les pauvres de la ville globale participent, de manière très modique et peu durable à la richesse qui est créée autour d'eux. 


\section{Références}

Allard, S. W., \& Small, M. L. (2013). Reconsidering the urban disadvantaged the role of systems, institutions, and organizations. The ANNALS of the American Academy of Political and Social Science, 647(1), 6-20.

Anderson, E. (1978). A Place on the Corner. A Study of Black Streetcorner Men. University of Chicago Press.

Anderson, E. (1990). Streetwise: Race, class, and change in an urban community. University of Chicago Press.

Anderson, E. (1999). Code of the Street. Decency, Violence, and the Moral Life of the Inner City. New York: Norton.

Anderson, E. (2011). The cosmopolitan canopy: Race and civility in everyday life. WW Norton \& Company.

Anderson, E. (2012). The iconic ghetto. The ANNALS of the American Academy of Political and Social Science, 642(1), 8-24.

Bearman, P. (2005). Doormen. University of Chicago Press.

Black, T. (2009). When a heart turns rock solid: The lives of three Puerto Rican brothers on and off the streets. New York: Pantheon Books.

Boissevain, J. (1966). Patronage in Sicily. Man, 1(1): 18-33.

Bonnet F. (2010), Les machines politiques aux États-Unis. Clientélisme et immigration entre 1870 et 1950 , Politix, ${ }^{\circ} 92$, pp. $7-27$

Bourgois, P. (1996). In search of respect: Selling crack in El Barrio. Cambridge University Press.

Carr, P. J. (2003). The new parochialism: The implications of the beltway case for arguments concerning informal social control. American Journal of Sociology, 108(6), 12491291.

Contreras, R. (2012). The stickup kids: Race, drugs, violence, and the American dream. University of California Press.

Desmond, M. (2012a). Disposable Ties and the Urban Poor. American Journal of Sociology, 117(5), 1295-1335.

Desmond, M. (2012b). Eviction and the Reproduction of Urban Poverty. American Journal of Sociology, 118(1), 88-133.

Desmond, M. (2014), Relational Ethnography, Theory and Society 43: 547-79.

Desmond, M., \& Valdez, N. (2013). Unpolicing the urban poor consequences of third-party policing for inner-city women. American Sociological Review, 78 (1): 117-141.

Drake, S. C., \& Cayton, H. (1945). Black Metropolis. 2 vols. New York: Harper\& Row.

Duneier, M. (1994). Slim's table: Race, respectability, and masculinity. University of Chicago Press.

Duneier, M. (1999). Sidewalk. New York : Macmillan.

Duvoux, N. (2010), "Repenser la culture de la pauvreté", La Vie des idées. http://www.laviedesidees.fr/Repenser-la-culture-de-la-pauvrete.html

Edin, K., Kefalas M. (2005). Promises I Can Keep. Why poor women put motherhood before marriage, Berkeley: University of California Press

Edin, K., Lein, L. (1997). Making ends meet: How single mothers survive welfare and lowwage work. New York: Russell Sage Foundation Publications.

Erie, S. P. (1990). Rainbow's end: Irish-Americans and the dilemmas of urban machine politics, 1840-1985. University of California Press. 
Gans, H. J. (1972). The positive functions of poverty. American Journal of Sociology, 78 (2): 275-289.

Garfinkel, H. (1956). Conditions of successful degradation ceremonies. American journal of sociology, 61 (5): 420-424.

Gieryn, T. F. (2000). A space for place in sociology. Annual review of sociology, vol. 26, pp. 463-496.

Goffman, A. (2014). On the run: Fugitive life in an American city. University of Chicago Press.

Goffman, E. (1959). The presentation of self in everyday life. New York: Double Day

Goffman, E. (1967). Interaction rituals. New York: Anchor Books.

Granovetter, M. (1985). Economic action and social structure: The problem of embeddedness, American Journal of Sociology, 91 (3): 481-510.

Halpern-Meekin Sarah, Kathryn Edin, Laura Tach, Jennifer Sykes. (2015). It's Not Like I'm Poor. How Working Families Make Ends Meet in a Post-Welfare World. University of California Press

Harding, D. J. (2010). Living the drama: Community, conflict, and culture among inner-city boys. University of Chicago Press.

Jargowsky, P. A. (1997). Poverty and place: Ghettos, barrios, and the American city. Russell Sage Foundation.

Jargowsky, P. A. (2015). Architecture of Segregation: Civil Unrest, the Concentration of Poverty, and Public Policy.The Century Foundation, New York, and the Center for Urban Research and Education, Rutgers-Camden, August 9, 2015

Katz, J. (1982). Poor people's lawyers in transition. Rutgers University Press.

Lamont, M. et Small, M.L. (2008). How Culture Matters : Enriching our Understandng of Poverty », Pp 76-102 in A.C. Lin et D.R. Harris (dir.), The Colors of Poverty, New York : Russell Sage Foundation

Lee, J. (2002). Civility in the city: Blacks, Jews, and Koreans in urban America. Harvard University Press.

Light, I., \& Bonacich, E. (1988). Immigrant Entrepreneurs: Koreans in Los Angeles, 19651982. University of California Press.

Marwell, N. P. (2004). Privatizing the welfare state: Nonprofit Community based organizations as political actors. American Sociological Review 69:265-91.

Marwell, N. P. (2007). Bargaining for Brooklyn: Community organizations in the entrepreneurial city. Chicago: University of Chicago Press.

Marwell, N. P., \& McQuarrie, M. (2013). People, place, and system organizations and the renewal of urban social theory. The ANNALS of the American Academy of Political and Social Science, 647(1), 126-143.

Massey, D. S., \& Denton, N. A. (1993). American apartheid: Segregation and the making of the underclass. Harvard University Press.

McQuarrie, M., \& Marwell, N. P. (2009). The missing organizational dimension in urban sociology. City \& Community, 8(3), 247-268.

Mollenkopf, J. H., \& Castells, M. (Eds.). (1991). Dual City: Restructuring New York. Russell Sage Foundation.

Murray Charles (1984), Losing ground. American Social Policy, 1950-1980, New York: Basic Books

Newman, K. S., \& Chen, V. T. (2007). The missing class: Portraits of the near poor in America. Beacon Press. 
Newman, K. S., \& Massengill, R. P. (2006). The texture of hardship: Qualitative sociology of poverty, 1995-2005. Annual Review of Sociology, 423-446.

Pattillo, Mary. (2007). Black on the Block. The Politics of Race and Class in the City. Chicago: Chicago University Press

Pattillo-McCoy, Mary. (1999). Black Picket Fences. Privilege and Peril among the Black Middle Class. Chicago: Chicago University Press

Pettit, B., \& Western, B. (2004). Mass imprisonment and the life course: Race and class inequality in US incarceration. American Sociological Review, 69(2), 151-169.

Portes A. et Sensenbrenner J. (1993). Embeddedness and immigration: Notes on the social determinants of economic action, American Journal of Sociology, 98 (6): 13201350

Quillian, L. (1999). Migration Patterns and the Growth of High-Poverty Neighborhoods, 1970-1990. American Journal of Sociology, 105(1), 1-37.

Sampson, R. J. (2012). Great American city: Chicago and the enduring neighborhood effect. University of Chicago Press.

Sassen, S. (1991). The Global City. New York, London and Tokyo, Princeton : Princeton University Press.

Scott, J. C. (1985). Weapons of the weak: Everyday forms of peasant resistance. Yale University Press.

Small, M. L. (2004). Villa Victoria. The transformation of social capital in a Boston barrio. Chicago: University of Chicago Press.

Small, M. L. (2008). Four reasons to abandon the idea of "The Ghetto". City \& community, 7(4), 389-398.

Small, M. L. (2009). Unanticipated gains. Origins of network inequality in everyday life. Chicago: University of Chicago Press.

Small, M. L., \& McDermott, M. (2006). The presence of organizational resources in poor urban neighborhoods: An analysis of average and contextual effects. Social Forces, 84(3), 1697-1724.

Small, M. L., \& Newman, K. (2001). Urban poverty after the truly disadvantaged: The rediscovery of the family, the neighborhood, and culture. Annual Review of sociology, 23-45.

Smith, S. S. (2007). Lone pursuit: Distrust and defensive individualism among the black poor. Russell Sage Foundation.

Thery, C. (2014). Larry's clique. The informal side of the housing market in low-income minority neighborhoods, $\mathrm{PhD}$. Dissertation, Columbia University

Venkatesh, S. A. (2006). Off the books: The underground economy of the urban poor. Cambridge, MA: Harvard University Press.

Venkatesh, S. A. (2008). Gang leader for a day: A rogue sociologist takes to the streets. Penguin.

Venkatesh, S.A. (2000). American Project. The Rise and Fall of a Modern Ghetto. Cambridge, MA: Harvard University Press

Venkatesh, S.A. (2013). Floating City. A Rogue Sociologist Lost and Found in New York's Underground Economy, Penguin.

Wacquant, L. (2001). Deadly symbiosis when ghetto and prison meet and mesh. Punishment \& Society, 3(1), 95-133.

Wacquant, L. (2002). Scrutinizing the Street: Poverty, Morality, and the Pitfalls of Urban Ethnography. American Journal of Sociology 107(6): 1468-1532

Wacquant, L. (2004). Body \& Soul. New York: Oxford University Press. 
Wacquant, L. (2009). Punishing the poor: The neoliberal government of social insecurity. Duke University Press.

Wacquant, L. (2011). "A Janus-Faced Institution of Ethnoracial Closure: A Sociological Specification of the Ghetto." Pp. 1-31 in Ray Hutchison and Bruce Haynes (eds.), The Ghetto: Contemporary Global Issues and Controversies, Boulder, Westview.

Wacquant, L. J. (1997). Three pernicious premises in the study of the American ghetto. International Journal of Urban and Regional Research, 21(2), 341-353.

Waldinger, R. (1996). Still the Promised City? New Immigrants and African-Americans in Post-Industrial New York, Cambridge, MA: Harvard University Press.

Waldinger, R., \& Lichter, M. I. (2003). How the other half works: Immigration and the social organization of labor. Univ of California Press.

Watkins-Hayes, C. (2009). The new welfare bureaucrats: Entanglements of race, class, and policy reform. University of Chicago Press.

Whyte, W. F. (1943). Street corner society: The social structure of an Italian slum. University of Chicago Press.

Wilson, W. J. (1987). The truly disadvantaged: The inner city, the underclass, and public policy. University of Chicago Press.

Wilson, W. J. (1996). When work disappears: The world of the new urban poor. Knopf.

Wolf, E. R. (1966). Kinship, friendship, and patron-client relations in complex societies. The social anthropology of complex societies, 1-22.

Young, A. (2004). The minds of marginalized black men. Princeton, NJ: Princeton University Press.

Zhou, M., \& Logan, J. R. (1989). Returns on human capital in ethnic enclaves: New York City's Chinatown. American sociological review, 54 (5) : 809-820. 